\title{
Effect of antibiotic treatment in preweaned Holstein calves after experimental bacterial challenge with Pasteurella multocida
}

\author{
C. L. Holschbach, S. M. Raabis, and T. L. Ollivett* \\ Department of Medical Sciences, School of Veterinary Medicine, University of Wisconsin-Madison 53706
}

\section{ABSTRACT}

The primary objective of this randomized controlled challenge study was to investigate the effect of ampicillin on ultrasonographic (US) lung consolidation after experimental challenge with Pasteurella multocida in preweaned dairy calves. The secondary objectives were to determine whether ampicillin affected respiratory score, gross consolidation, or the detection of $P$. multocida in lung tissue at postmortem exam (PME). Holstein bull calves $(\mathrm{n}=39)$ were transported to the University of Wisconsin-Madison School of Veterinary Medicine isolation facility at the mean $( \pm \mathrm{SD})$ age of 52 $\pm 6 \mathrm{~d}$. After a 7-d acclimation period, 30 calves were inoculated intratracheally with $10^{10} \mathrm{cfu}$ of ampicillinsensitive $P$. multocida. Lung US and respiratory scoring were performed $2,6,12$, and $24 \mathrm{~h}$ post-challenge, then US once daily and respiratory scoring twice daily until $\mathrm{d}$ 14. Calves were randomized to receive ampicillin [n $=17$, treatment $(\mathrm{TX}), 6.6 \mathrm{mg} / \mathrm{kg}$ i.m. once daily for 3 d] or placebo $[n=11$, control $(\mathrm{CON})$, saline, equal volume, i.m. once daily for $3 \mathrm{~d}$ ] when $\geq 1 \mathrm{~cm}^{2}$ of lung consolidation was observed and $\geq 6 \mathrm{~h}$ had elapsed since challenge. Lung lesions $\geq 1 \mathrm{~cm}^{2}$ were considered positive for consolidation. Calves were respiratory score positive if $\geq 2$ in 2 or more categories based on the Wisconsin respiratory health score chart. Area under the curve (AUC) was calculated for US score and respiratory score as a proxy for time with consolidation and clinical respiratory disease, respectively. Gross lung lesions and pathogens were quantified following PME. At the time of first treatment, consolidation had developed in $28 / 30$ calves ( $\mathrm{TX}, \mathrm{n}=17 ; \mathrm{CON}, \mathrm{n}=11)$ and $6 \%(1$ out of 17) of TX and $9 \%$ (1 out of 11) of CON calves had a positive respiratory score. The TX calves had a significantly lower median (interquartile range given in parentheses) AUC for US score [TX: $23(20,29)$, CON: $47(33,53)]$, whereas mean AUC for respiratory score was not different between groups (TX: $93 \pm 28$, CON:

Received May 20, 2019.

Accepted August 5, 2019.

*Corresponding author: theresa.ollivett@wisc.edu
$96 \pm 17$ ). On d $14,70 \%$ (12 out of 17 ) of TX and $100 \%$ (11 out of 11) of CON calves had lung consolidation, and $24 \%$ (4 out of 17) of TX and $27 \%$ (3 out of 11) of $\mathrm{CON}$ calves had clinical respiratory disease. On PME, median consolidation was $10 \%(6,15)$ for TX and $10 \%$ $(2,28)$ for CON calves. Lung cultures were positive for P. multocida in $77 \%$ (13 out of 17 ) of TX and $91 \%$ (10 out of 11) of CON calves. Lung health benefited from a 3-d ampicillin therapy, but benefits were short-lived. Treatment failures might be due to incomplete resolution of the initial lung infection. Future studies are needed to optimize TX strategies to improve long-term lung health.

Key words: bovine respiratory disease, Pasteurella multocida, ampicillin, lung ultrasound

\section{INTRODUCTION}

Respiratory disease in adult dairy cattle is reported infrequently by producers ( $<3 \%$; USDA, 2018). However, $13 \%$ of condemnations in dairy cull cows are due to pneumonia, making it the second leading cause of postmortem condemnations (White and Moore, 2009). According to the latest National Animal Health Monitoring System dairy study, $95 \%$ of adult cows with producer-diagnosed respiratory disease were treated with antibiotics, the vast majority receiving a $\beta$-lactam (USDA, 2018). Interestingly, nearly $40 \%$ of affected cows were removed from the herd despite antibiotic therapy in most cases (USDA, 2018). Treatment failures due to late detection or incorrect antibiotic dose, duration, spectrum, or bacterial resistance are potential reasons for poor outcomes after therapy.

Purulent nasal discharge, cough, poor milk production, and weight loss usually lead to the diagnosis of chronic suppurative bronchopneumonia; however, the response to treatment is rarely assessed quantitatively (Barrett, 2000; Scott, 2013). Auscultation failed to identify the nature and extent of lung pathology in adult cattle with chronic suppurative pneumonia, whereas ultrasonographic (US) examination of the chest provided critical information in the diagnosis of suppurative pulmonary disease and in formulating a prognosis (Scott, 2013). 
Bacteria such as Trueperella pyogenes are commonly found within lungs of affected cattle, potentially walled off and inaccessible to antibiotics (Barrett, 2000). As a result, these animals may respond poorly to treatment, suffer chronic weight loss, or even die as the result of an acute exacerbation of respiratory disease (Barrett, 2000). In a more recent report, favorable responses to long-term penicillin therapy in 8 of 12 cows with US evidence of suppurative bronchopneumonia challenges the idea that all cattle with chronic pneumonia respond poorly to treatment (Scott, 2013).

Currently, relatively few antibiotic treatment options exist for lactating dairy cattle with respiratory disease. Although macrolide, phenicol, and fluoroquinolone products are commonly used for treating nonlactating cattle, none of these are approved for use in lactating dairy cattle (female cattle 20 mo of age or older). Conversely, ampicillin trihydrate (Polyflex, Boehringer Ingelheim, Duluth, GA) has FDA approval for the treatment of respiratory disease in lactating dairy cattle. Ampicillin provides bactericidal activity against gram-positive and gram-negative organisms, more specifically Pasteurella multocida, a common bacterial agent associated with bovine respiratory disease (BRD; Dabo et al., 2007).

The aim of this study was to improve our understanding of bovine lung response to antibiotic therapy after a bacterial respiratory infection, by assessing lung consolidation via US and gross postmortem examination (PME) and clinical response using respiratory scoring. Ultrasound can evaluate the extent of lung consolidation and is a reliable method of confirming bronchopneumonia in calves with or without clinical signs of BRD (Ollivett and Buczinski, 2016). An additional aim of the study was to determine whether antibiotic treatment would alter the likelihood of isolating bacterial pathogens in lung tissue at PME. Ampicillin trihydrate was selected because of its FDA approval status and frequent use in lactating dairy cattle with respiratory disease. Given the relatively low rates of clinical respiratory disease in lactating cattle, a challenge model using calves could provide information regarding lung response to therapy in a more efficient and cost-effective manner than a field trial in lactating cattle.

Our first objective was to investigate the effect of ampicillin on US lung consolidation after experimental challenge with $P$. multocida in preweaned dairy calves. The secondary objectives were to determine whether ampicillin affected respiratory score, gross consolidation, or detection of $P$. multocida in lung tissue at PME. Our hypotheses were that treatment with ampicillin would decrease the proportion of calves that developed severe lung consolidation and clinical respi- ratory disease after experimental infection, compared with controls. Additionally, the treated group would have less gross consolidation and fewer calves with $P$. multocida isolated from lung tissue at PME compared with controls.

\section{MATERIALS AND METHODS}

\section{General}

This randomized controlled study was completed between July 11, 2017, and August 2, 2017, at the University of Wisconsin Livestock Laboratory in Madison, Wisconsin. This research project was approved by the University of Wisconsin Institutional Animal Care and Use Committee (protocol \#A005636-A02) and the Institutional Biosafety Committee (protocol \#B00000610).

\section{Animals and Experimental Groups}

Forty-five Holstein bull calves, born between May 12, 2017, and May 30, 2017, were raised in individual calf hutches at a commercial dairy in southeast Wisconsin. All calves received colostrum or colostrum replacer following birth and had blood drawn for serum total protein measurement via digital refractometry between $24 \mathrm{~h}$ and $6 \mathrm{~d}$ of age. Additionally, all calves were administered an intranasal respiratory vaccine (Inforce 3, Zoetis, Kalamazoo, MI) during the first $24 \mathrm{~h}$ of life. Respiratory scoring (McGuirk and Peek, 2014) and lung US (Ollivett and Buczinski, 2016) were performed once weekly for 3 weeks before transport to the research facility to monitor for respiratory disease.

Clinical respiratory scoring assigned 0 to 3 points for each of the following categories: rectal temperature, nasal discharge, cough, ocular discharge, and ear positioning (McGuirk and Peek, 2014). Calves with respiratory scores $\geq 2$ in 2 or more categories were considered positive (clinical). Fever was defined as a temperature score $3\left(\geq 39.4^{\circ} \mathrm{C}\right)$, and high fever was a temperature $\geq 40^{\circ} \mathrm{C}$. Regarding lung US, left and right lung fields were scanned, and images were interpreted using a 0 to 5 US lung score, as previously described (Ollivett and Buczinski, 2016). An US score 0 indicates normal aerated lung, with no consolidation and minimal comet-tail artifact (Ollivett and Buczinski, 2016). A score 1 signifies diffuse comet-tail artifact without consolidation (Ollivett and Buczinski, 2016). When $\geq 1$ $\mathrm{cm}^{2}$ of lung consolidation develops, an US score of 2 is assigned (Ollivett and Buczinski, 2016). Scores of 3, 4, and 5 represent 1,2 , and 3 or more completely consolidated lung lobes, respectively (Ollivett and Buczinski, 2016).Calves received the score corresponding to their 
most significant lesion. The depth and dorsal-to-ventral extent of consolidated lung were measured within each intercostal space using the 1-cm grid lines on the US screen (Ibex Pro, E. I. Medical, Loveland, CO). For the purpose of this study, a normal lung US was score 0 or $1 ; \geq 1 \mathrm{~cm}^{2}$ consolidation, a score $\geq 2$; and severe consolidation, a score $\geq 3$. Of the 45 calves, 39 remained respiratory score negative with a normal lung US and did not receive treatment for diarrhea or respiratory disease, thus meeting inclusion criteria for the study. These calves $(n=39)$ were transported from the source farm to the university isolation facility at the mean \pm standard deviation (SD) age of $52 \pm 6$ d. Upon arrival, the calves were weighed and had 2 deep nasopharyngeal swabs (DNPS) collected for respiratory pathogen analysis. One swab was placed in M6 viral transport medium, and the other in Amies bacterial transport medium. All DNPS swabs were submitted to the Wisconsin Veterinary Diagnostic Laboratory (WVDL, Madison) for bovine complete respiratory panels.

\section{Calf Housing and Feeding Management}

Calves $(\mathrm{n}=39)$ were identified with unique numerical plastic ear tags and randomly allocated to 1 of 4 pens ( 3 pens of 10 and 1 pen of 9 ), using a random number generator. All pens were located in a single room with concrete walls and slatted floors. Pens 1 and 4, as well as 2 and 3 , were adjacent to each other. The pens were divided by a solid partition, preventing physical contact between calves in different pens. Pens were cleaned and disinfected twice daily by university staff.

Calves were fed milk replacer (Land O'Lakes Amplifier Max 22:20, $12 \mathrm{oz}$. powder to 2 quarts water; Arden Hills, MN) every $12 \mathrm{~h}$ via a milk bar. Each pen was equipped with an automatic waterer, and fresh freechoice texturized calf starter (Vita Plus, $18 \%$ crude protein; Madison, WI) was provided daily.

\section{Experimental Design and Data Collection}

A visual timeline of data collection is portrayed in Figure 1. After a 7-d acclimation period, 30 calves were enrolled in the challenge study. Enrollment criteria included a temperature $\leq 39.4^{\circ} \mathrm{C}$, normal lung US, and a negative respiratory score. Enrollment took place the day before challenge (baseline, $\mathrm{d}-1$ ), at which time a second set of DNPS were collected from each calf. Of 39 calves, 33 met enrollment criteria. One calf each from pens 1 through 3 , and 3 calves from pen 4 , did not meet enrollment criteria. To keep pen numbers similar and prevent additional stress from rearranging social groups, numbers were drawn from a hat to randomly eliminate an additional calf from pens 1 through 3 . Final pen compositions included 3 pens of 8 and one pen of 6 .

The challenge solutions were developed using a wild-type strain of P. multocida A1 obtained from the WVDL. The strain of $P$. multocida used in the study was considered sensitive (MIC $\leq 0.2500)$ to ampicillin.

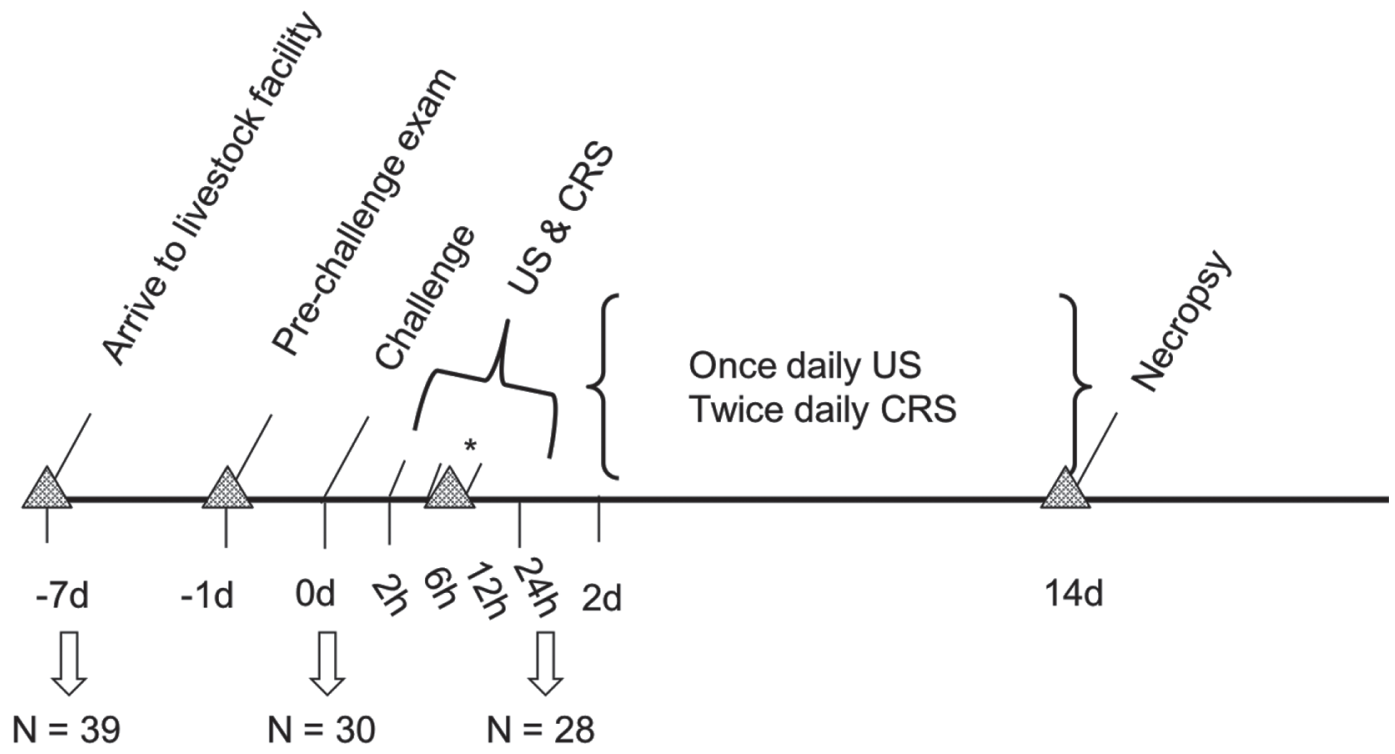

Figure 1. Challenge study timeline. Triangles represent timing of deep nasopharyngeal swab collection. The first swabs were collected upon arrival to the livestock laboratory, $7 \mathrm{~d}$ before challenge. The second set of swabs was collected before challenge on $\mathrm{d}-1$. The third set was collected at the time of the calf's first treatment, which occurred when at least $1 \mathrm{~cm}^{2}$ of consolidation was observed and $\geq 6 \mathrm{~h}$ had elapsed since challenge (denoted by asterisk). The fourth set of swabs was collected following final examination on d 14 , before euthanasia. US $=$ lung ultrasound; CRS = clinical respiratory score. 
Individual tubes containing $10 \mathrm{~mL}$ of brain heart infusion broth were inoculated with $P$. multocida. Tubes were incubated at $37^{\circ} \mathrm{C}$ for 18 to $24 \mathrm{~h}$. After growth occurred, the tubes were centrifuged to pellet the bacterial cells. The pellet in each tube was resuspended in $10 \mathrm{~mL}$ PBS with $5 \%$ fetal bovine serum (FBS) to create a solution containing $P$. multocida at $10^{9} \mathrm{cfu} / \mathrm{mL}$ in $10 \mathrm{~mL}$ PBS $+5 \%$ FBS, for a total dose of $10^{10} \mathrm{cfu} /$ calf. The FBS was prepared by adding $20 \mathrm{~mL}$ FBS to $380 \mathrm{~mL}$ PBS. One tube was later plated and confirmed the $\mathrm{cfu} / \mathrm{mL}$ in the inocula. Just before inoculation, each dose was extended to $25 \mathrm{~mL}$ by adding $15 \mathrm{~mL}$ of PBS.

On challenge day (d 0 ), calves were sedated with $0.05 \mathrm{mg} / \mathrm{kg}$ xylazine i.v. (AnaSed injection, $20 \mathrm{mg} / \mathrm{mL}$; Akorn Animal Health, Lake Forest, IL). A rigid multifunction digital endoscope (iVetscope, S.E.C Repro Inc., Quebec, Canada) was used to visualize the oropharynx, the arytenoids, and the subsequent passage of a sterile equine infusion pipette through the arytenoids into the trachea (Equine Infusion Pipette by Continental Plastics, distributed by Valley Vet Supply, Marysville, KS). The pipette was advanced to the level of the thoracic inlet, just proximal to the tracheal bifurcation, and a $25-\mathrm{mL}$ suspension of $10^{10} \mathrm{cfu}$ of ampicillin-sensitive P. multocida was instilled. Immediately following instillation of the inoculum, calves were stimulated to take deep breaths by partially occluding the nares for $5 \mathrm{~s}$. Lung US and respiratory scoring were performed $2,6,12$, and $24 \mathrm{~h}$ post-challenge, then US once daily and respiratory scoring twice daily until d 14 . In total, calves underwent 18 US exams and 31 respiratory scores. Respiratory scoring was performed by blinded research assistants before lung US. To decrease interobserver variability, all scorers were trained by the same person $(\mathrm{CH})$. Ultrasonography was performed by 2 experienced operators ( $\mathrm{CH}$ and $\mathrm{SR}$ ). Observations and measurements were spoken and recorded on an iPad by a research assistant. The ultrasonographers $(\mathrm{CH}$ and SR) were blinded to treatment group throughout all data collection.

Calves were randomized to receive $250 \mathrm{mg} / \mathrm{mL}$ ampicillin $[\mathrm{n}=17$, treatment $(\mathbf{T X}): 6.6 \mathrm{mg} / \mathrm{kg}$ i.m. once daily for $3 \mathrm{~d}]$ or placebo $[\mathrm{n}=11$, control $(\mathbf{C O N})$ : sterile saline, equal volume, i.m. once daily for $3 \mathrm{~d}$ ] using a random number generator. Treatment began when at least $1 \mathrm{~cm}^{2}$ of consolidation was observed and $\geq 6 \mathrm{~h}$ had elapsed since challenge. A third set of DNPS were collected before treatment. Following the final examination on d 14, a fourth set of DNPS were collected, and calves were euthanized with an intravenous overdose (1 mL/10 lbs) of barbiturate (Beuthanasia-D Special, Merck Animal Health, Kenilworth, NJ). Sixteen calves were euthanized on challenge d 14 , and the remaining 14 were euthanized on challenge d 15 . Calves were chosen for euthanasia based on the order in which they were inoculated.

A PME was performed immediately following euthanasia. Digital photographs were taken of lungs in situ (calves in left lateral recumbency); then the lungs and heart were removed en bloc from the thoracic cavity, cleaned, and all lesions noted. Digital photos were taken of the right and left lung from the lateral perspective, for later morphometric analysis, and individual lung lobe and heart weights were obtained. Lung-to-heart weight ratios were used as an indicator of the degree of inflammatory infiltrates in the lung, with the assumption that lungs with less consolidation and therefore fewer infiltrates would weigh less and have a smaller lung-to-heart ratio than lungs with more severe infiltrates (J. L. Caswell, University of Guelph, Ontario, $\mathrm{CA}$; personal communication). These ratios were calculated by summing the individual lung weights and dividing by the heart weight. Representative tissue samples were collected for bacteriology.

Bovine respiratory disease complete panels were performed at the WVDL on 4 sets of DNPS and lung tissue samples. The PCR tests included in this panel are bovine viral diarrhea virus, infectious bovine rhinotracheitis virus, bovine syncytial virus, bovine respiratory coronavirus (BoCV), Bibersteinia trehalosi, Histophilus somni, Mannheimia haemolytica, Pasteurella multocida, and Mycoplasma bovis. Bacterial culture and antimicrobial susceptibility were also performed. Real-time PCR provides a relative value cycle threshold $(\mathbf{C T})$, which indicates the amount of target nucleic acid in the sample. Cycle threshold is inversely proportional to the amount of target present in the sample (i.e., lower CT values indicate more nucleic acid). Calves were considered PCR positive for the aforementioned pathogens when the CT value was $<40$, a cutoff determined by the WVDL.

Morphometric analysis was used to quantify the percent surface area of consolidated lung on gross PME. Using a computer program (ImageJ, Laboratory for Optical and Computational Instrumentation, Madison, WI), each lung lobe was outlined to determine the total surface area of that particular lung. Then, each area of consolidation was outlined and summed to ascertain the total surface area of consolidation. The combined surface area of consolidation for both lungs was divided by the total surface area of both lungs to determine the overall percent consolidation.

\section{Statistical Analyses}

The experimental unit was the individual calf. Based on previous work (Ollivett, 2014) and clinical experience, we based our sample size calculation on the as- 
sumption that $80 \%$ of challenged, untreated (CON) calves and $10 \%$ of challenged, antibiotic-treated (TX) calves would be affected by severe lung consolidation. We planned to enroll 2 TX calves for every CON calf, which would result in approximately 20 TX and 10 CON calves $(\beta=0.90)$.

A standard statistical package was used for all analyses (SAS v. 9.4, SAS Institute Inc., Cary, NC). After graphing US score (y-axis) by time point (x-axis), area under the curve (AUC) was determined for each calf by summing the trapezoidal area between successive time points. Individual AUC were summarized for each group and reported as median and interquartile range (given in parentheses). After graphing total respiratory score (y-axis) by time point (x-axis), the same approach was taken to calculate AUC for clinical respiratory score. All continuous variables were assessed for normality using the Shapiro-Wilk test. Variables that followed a normal distribution, AUC for clinical respiratory score, maximum total respiratory score, and lung-to-heart weight ratio, were compared using Student's t-test, and the measure of central tendency was reported as mean and SD. Group-level comparisons for continuous variables that did not follow a normal distribution were evaluated using the Wilcoxon rank sum test, and measures of central tendency were reported as median and interquartile range. These variables were number of US exams with consolidation, number of US exams with severe consolidation, AUC for US score, exam number at first positive respiratory score following challenge, number of positive respiratory score exams, US exam number when calves first met treatment criteria, lung US score at first treatment, total respiratory score at first treatment, number of exams with fever $\geq 39.4^{\circ} \mathrm{C}$, maximum total respiratory score, and percent consolidation on morphometric analysis. Group-level comparisons of categorical variables were assessed with contingency tables and chi-squared analysis, or Fisher's exact test when expected cell counts were $<5$. Significance was set at $\alpha \leq 0.05$.

\section{RESULTS}

All challenged calves $(\mathrm{n}=30)$ had adequate passive transfer of maternal antibody (serum total protein $\geq 5.5 \mathrm{~g} / \mathrm{dL}$; Tyler et al., 1996) and survived the study period. Twenty-eight calves developed at least $1 \mathrm{~cm}^{2}$ of lung consolidation within $24 \mathrm{~h}$ of challenge and were randomized to treatment $(\mathrm{TX}, \mathrm{n}=17 ; \mathrm{CON}, \mathrm{n}=11$ ). The challenge model failed to induce bronchopneumonia in 2 calves. These 2 calves were excluded from all analyses. Ultrasound score, clinical respiratory score, and exam number at the time of first treatment were similar between TX and CON calves, indicating suc- cessful randomization (Table $1, P>0.40$ ). Box and whisker plots demonstrating the distribution of US and clinical respiratory scores over the course of the study period are shown in Figure 2.

\section{Ultrasonographic Outcomes}

All calves had normal lung US scores before challenge (Figure 2). At the last treatment, lung US scores were similar between groups [TX: $3(2,4), \mathrm{CON}: 3(3$, 4); $P=0.46]$ The TX calves had fewer exams with consolidation and a smaller AUC for US score compared with CON calves (Table $2, \mathrm{P}<0.03$ ). Nearly all calves developed severe consolidation at least once [TX: $88 \%$ (15 out of 17 ), CON: $82 \%$ (9 out of 11 ); $P=1.00$ ]; and for affected calves, severe consolidation was detected 24 $(12,24)$ and $12(12,12)$ hours after inoculation for TX and CON calves, respectively $(P=0.20)$.

After treatment was initiated, a normal lung score (US score $=0$ or 1 ) was observed at least once for $71 \%$ (12 out of 17 ) and $27 \%$ (3 out of 11) of TX and CON calves, respectively $(P=0.05)$. Lung consolidation recurred in the latter half of the study (Figure 3). More specifically, of the calves that developed a normal lung score following treatment, lung consolidation recurred in $58 \%$ ( 7 out of 12 ) and $100 \%$ (3 out of 3 ) of TX and CON calves, respectively. At the endpoint of the study, $30 \%$ (5 out of 17 ) of TX and $0 \%$ ( 0 out of 11 ) of CON calves had normal lung scores $(P=0.12)$. Throughout the study period, the pattern of US lung consolidation did not match the pattern of positive clinical respiratory scores (Figure 3 ).

\section{Clinical Respiratory Outcomes}

Before challenge, all calves had normal clinical respiratory scores (Figure 3). At the time of first treatment, $6 \%$ (1 out of 17 ) and $9 \%$ (1 out of 11 ) of TX and CON calves, respectively, had positive clinical respiratory scores $(P=1.00)$. However, positive clinical respiratory scores and fever $\left(>39.4^{\circ} \mathrm{C}\right)$ eventually developed at least once in all calves and occurred similarly between groups (Table $3, \mathrm{P}>0.36$ ). In addition, depressed at-

Table 1. Clinical outcomes at time of first treatment by experimental treatment group; results reported as median (interquartile range)

\begin{tabular}{lccc}
\hline Outcome & Antibiotic & Control & P-value \\
\hline EXAM $_{\text {treat }}{ }^{1}$ & $4(4,4)$ & $4(4,4)$ & 0.40 \\
USS $_{\text {treat }}{ }_{3}$ & $2(2,3)$ & $3(2,3)$ & 0.90 \\
TRS $_{\text {treat }}$ & $3(3,5)$ & $3(3,4)$ & 0.78 \\
\hline
\end{tabular}

${ }^{1}$ Ultrasound exam number when calves first met treatment criteria.

${ }^{2}$ Lung ultrasound score at first treatment.

${ }^{3}$ Total respiratory score at first treatment. 
titude scores were detected at least once in $35 \%$ (6 out of 17 ) and $36 \%$ (4 out of 11) of TX and CON calves, respectively. Depression occurred more than once in only 2 calves. At the endpoint of the study, $76 \%$ (13 out of 17) of TX and $73 \%$ (8 out of 11) of CON calves had normal clinical respiratory scores $(P=1.00)$.

\section{Gross Postmortem Outcomes}

On PME, all lungs contained firm, dark purple to red gross lesions in the cranioventral lung lobes (Figure $4 \mathrm{a}$ ), and purulent exudate was commonly detected in bronchi and bronchioles. Fibrinous or fibrous adhesions to the thoracic body wall (Figure 4b), distended interlobular septa with edema or fibrin, and abscesses were variable findings. The mean lung-to-heart weight ratio was $2.5 \pm 0.36$ and $2.5 \pm 0.58$ for TX and CON calves, respectively $(P=0.70)$; and median percent consolida- tion was $10 \%(6,15)$ for TX and $10 \%(2,28)$ for CON calves $(P=0.97)$.

\section{Pathogen Outcomes}

Antemortem culture and PCR results from DNPS samples are described in Tables 4 and 5, respectively. Regardless of testing method, antibiotic treatment was not associated with pathogen detection within individual time points $(P>0.12)$. Regardless of treatment group, the risk of detecting P. multocida by DNPS was at least 7 times higher at the endpoint of study (DNPS4) compared with the prechallenge sample (DNPS2; culture: $P<0.01$; PCR: $P<0.04)$. In contrast, detection of $M$. haemolytica (by culture and PCR) and T. pyogenes (by culture only) was similar between the prechallenge and end-of-study DNPS samples $(P>0.46)$. Postmortem lung tissue culture and PCR results are described in
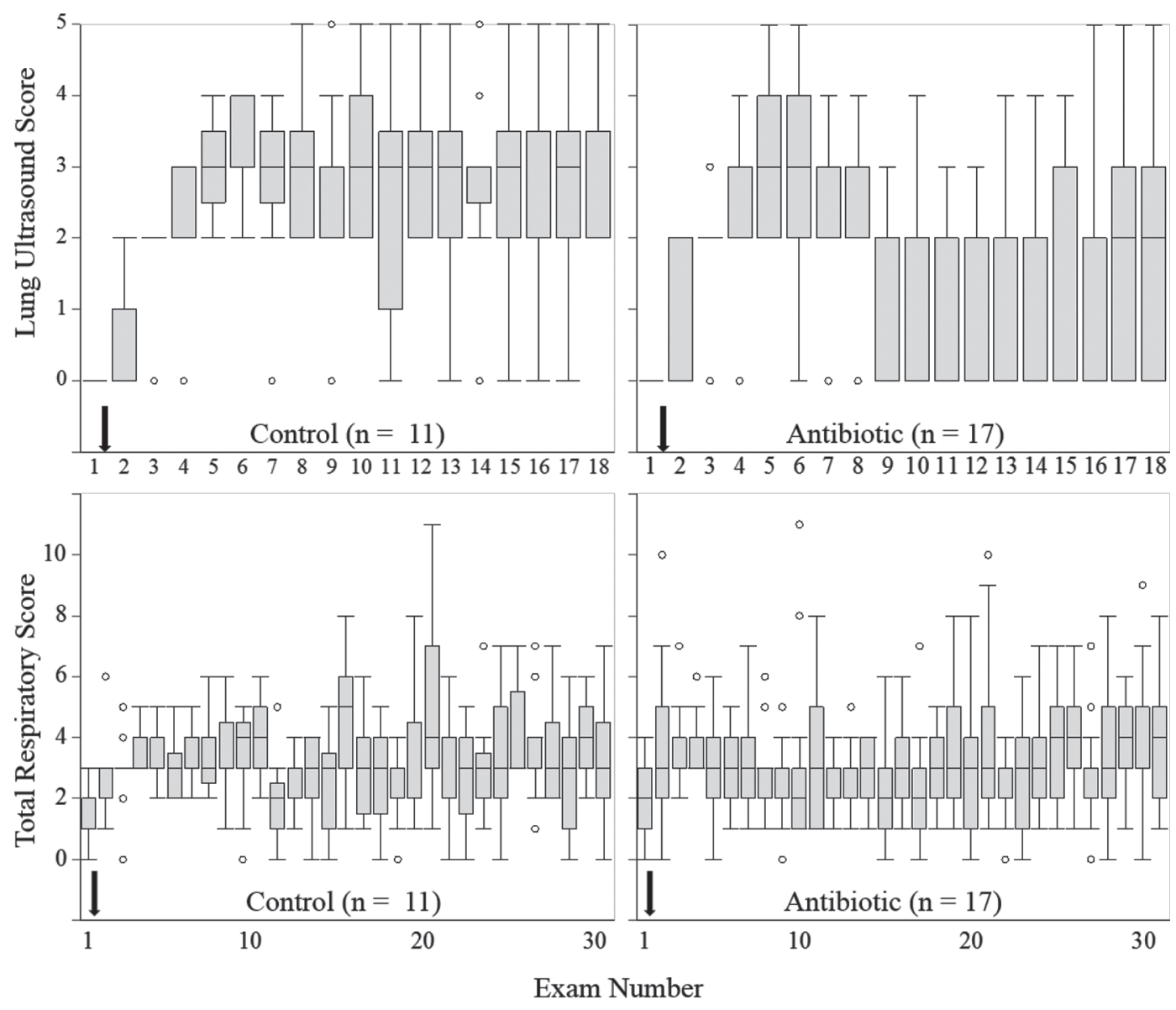

Figure 2. Boxplots of ultrasound scores and total respiratory scores at each examination throughout the study period. The line within each box is the median ultrasound score; the lines forming the bottom and top of each box are the 25th and 75th percentiles, respectively (interquartile range). Whiskers represent the minimum and maximum data points that fall within the range of $1.5 \times$ the interquartile distance below the lower quartile and above the upper quartile. Outliers are represented by open circles. The black arrows indicate when experimental challenge using Pasteurella multocida occurred. 
Table 2. Lung ultrasound outcomes by experimental treatment group; results reported as median (interquartile range)

\begin{tabular}{lccr}
\hline Outcome & Antibiotic & Control & $P$-value \\
\hline${\text { USS } 2+{ }^{1}}^{1}$ & $11(9,15)$ & $16(16,16)$ & 0.02 \\
${\text { USS } 3+{ }^{2}}^{2}$ & $3(2,8)$ & $12(9,15)$ & $<0.01$ \\
AUC $_{\text {uss }}{ }^{2}$ & $23(20,29)$ & $47(33,53)$ & 0.03 \\
\hline
\end{tabular}

${ }^{1}$ Number of ultrasound exams with consolidation.

${ }^{2}$ Number of ultrasound exams with severe consolidation.

${ }^{3}$ Area under the curve for ultrasound score.
Table 3. Clinical respiratory score outcomes by experimental treatment group; results reported as mean $\pm \mathrm{SD}$ or median (interquartile range)

\begin{tabular}{lccc}
\hline Outcome & Antibiotic & Control & $P$-value \\
\hline MAX $_{\mathrm{TRS}^{1}}{ }^{1}$ & $6.7 \pm 2.0$ & $7.4 \pm 1.6$ & 0.36 \\
AUC $_{\mathrm{CRS}}{ }^{2}$ & $93 \pm 28$ & $96 \pm 17$ & 0.77 \\
CRS+ ${ }_{\text {exam }}{ }_{4}$ & $7(2,19.5)$ & $10(5,16)$ & 0.36 \\
CRS ${ }_{\text {num }}{ }_{5}$ & $3(2,6)$ & $5(3,8)$ & 0.49 \\
Fever $+_{\text {num }}$ & $6(5,11)$ & $9(7,13)$ & 0.17 \\
\hline
\end{tabular}

${ }^{1}$ Maximum total respiratory score.

${ }^{2}$ Area under the curve for clinical respiratory score.

${ }^{3}$ Exam number at first positive respiratory score following challenge.

${ }^{4}$ Number of positive respiratory score exams.

${ }^{5}$ Number of exams with fever $\geq 39.4^{\circ} \mathrm{C}$.

A
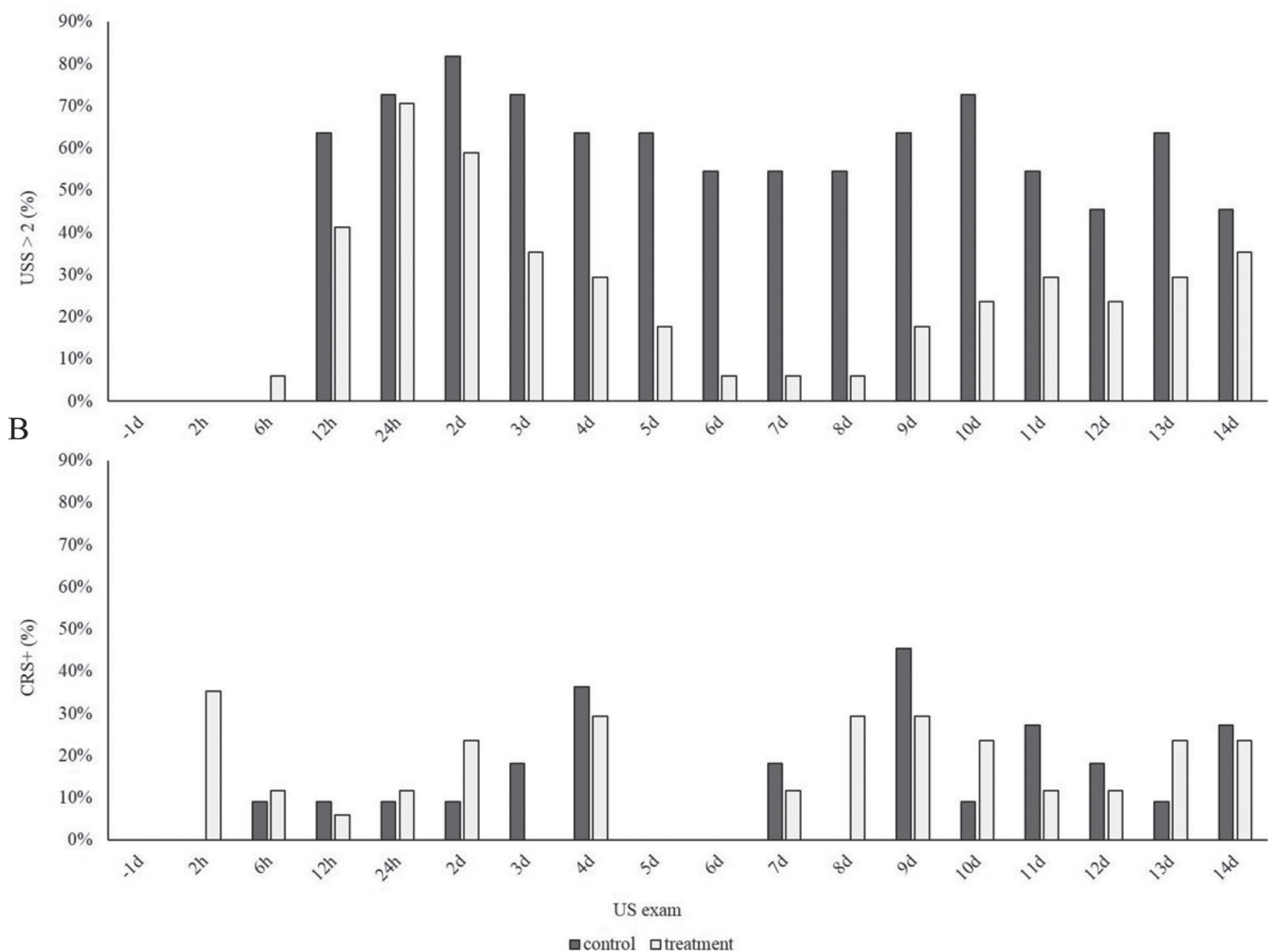

Figure 3. Proportion of calves with lobar pneumonia diagnosed by ultrasonographic score greater than 2 (USS; A) and positive clinical respiratory scores $(\mathrm{CRS}+$; B) throughout the study period. Exams were performed on the day before challenge $(-1 \mathrm{~d})$, and 2,6 , and $12 \mathrm{~h}$ postchallenge, then once daily until d 14 of the study. Calves were randomized to receive ampicillin ( $\mathrm{TX} ; \mathrm{n}=17 ; 6.6 \mathrm{mg} / \mathrm{kg}$ i.m. once daily for $3 \mathrm{~d}$ ) or placebo (CON; $\mathrm{n}=11$; saline, equal volume, i.m. once daily for $3 \mathrm{~d}$ ) when $\geq 1 \mathrm{~cm}^{2}$ of lung consolidation was observed and $\geq 6 \mathrm{~h}$ had elapsed since challenge. Calves were challenged intratracheally with $10^{10} \mathrm{cfu}$ of Pasteurella multocida. Dark gray bars = CON; light gray bars = TX. 
A

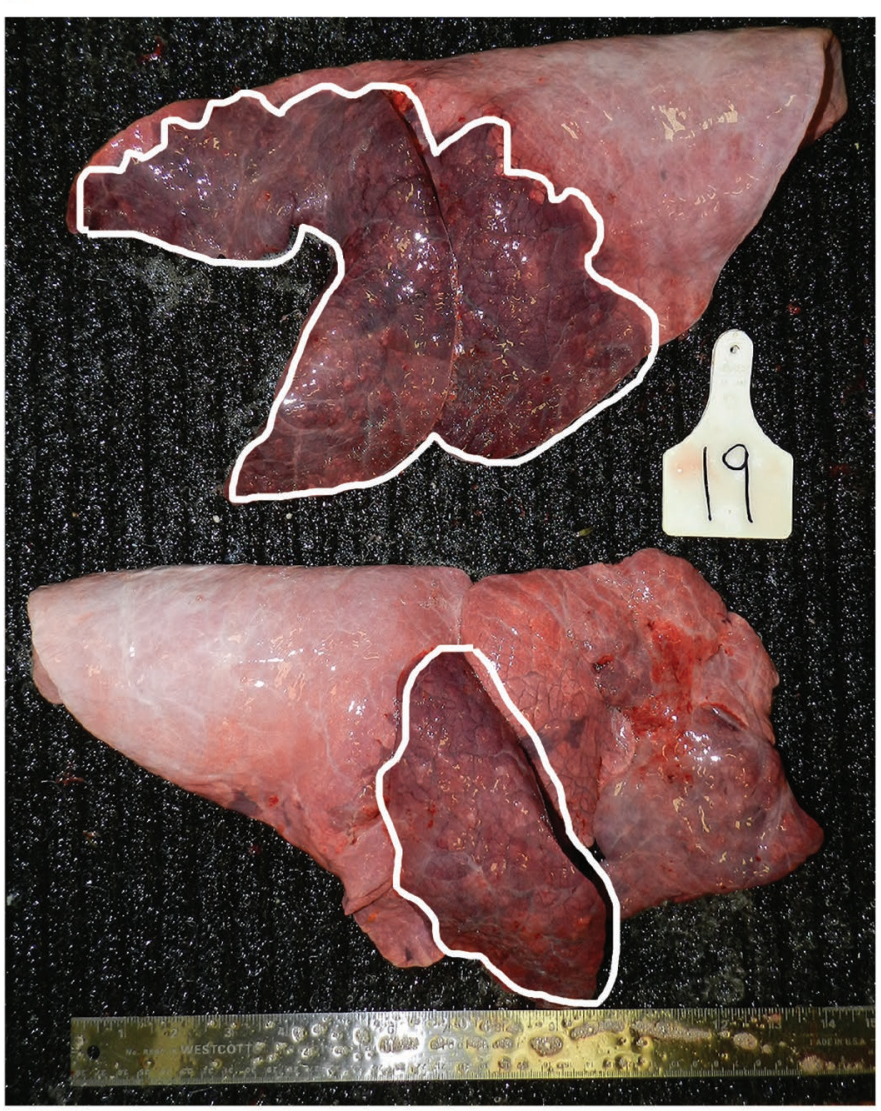

B

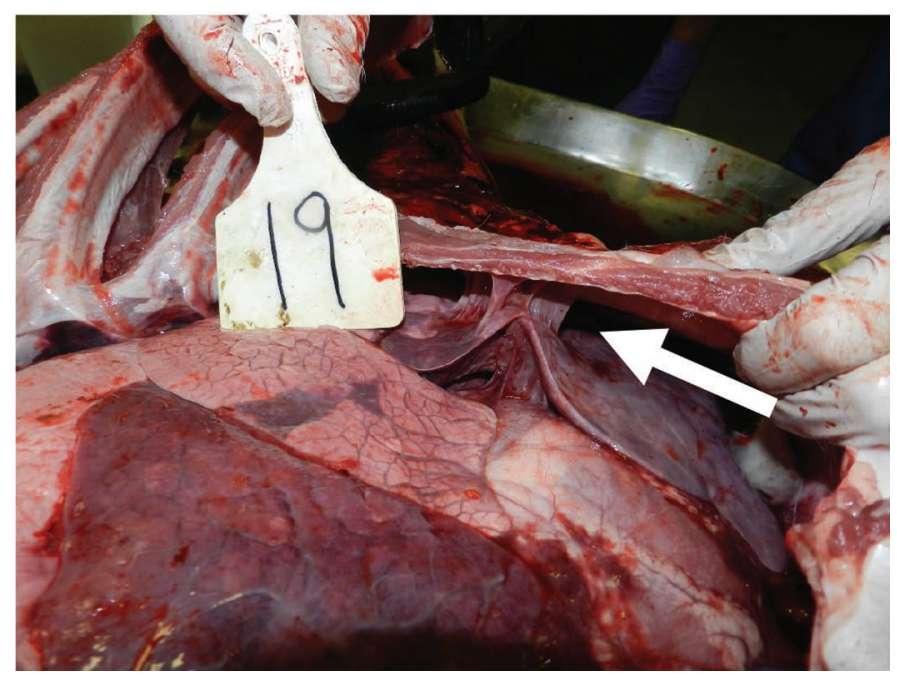

Figure 4. (A) Typical cranioventral distribution of consolidation in the left lung (top, white outline), and lobar consolidation of the right middle lung lobe (bottom, white outline). (B) In situ specimen. Carcass is in left lateral recumbency. The right 4 th rib is being reflected dorsally, exposing a fibrous adhesion between the lung and thoracic body wall (arrow).
Tables 6 and 7, respectively. For P. multocida PCRpositive samples (on lung tissue), the median $\mathrm{CT}$ values were $27.8(24,31.6)$ and $32(29.4,36.6)$ for $\mathrm{CON}$ and TX calves, respectively $(P=0.13)$.

Although all calves were negative upon arrival and the day before challenge, BoCV was eventually detected by PCR in both DNPS and lung tissue samples (Tables 5 and 7). More specifically, BoCV was detected in more calves at the end of the study (DNPS4) compared with prechallenge samples (Table 5; $P<0.003$ ). In the lung, BoCV was isolated (by PCR) less frequently among TX calves compared with CON calves (Table 7; $P=0.05$ ).

\section{DISCUSSION}

In this study, we successfully induced $P$. multocida bronchopneumonia and found that early antibiotic therapy can affect severity of lung consolidation in preweaned dairy calves. We also observed that lung consolidation can recur and pathogens can be detected in the lung and upper airway following treatment, even though the majority of calves were not clinical for respiratory disease during their last antibiotic treatment or at the end of the study. These results suggest that a possible mechanism for treatment failure in cattle is failure to completely cure the bacterial infection and associated lung consolidation.

The challenge model used in this study is intended to create acute, moderate to severe disease. The pathology was typical of cranioventral bacterial bronchopneumonia and consistent with gross reports from other studies (Dabo et al., 2007; Smith, 2015). As in our study, a previous $P$. multocida challenge study using a comparable technique found that depressed attitude gave very little evidence of severity of lung consolidation at PME, and, although rectal temperature and respiratory rates were higher in challenged animals, this information provided little indication of the extent of the pathological changes (Dagleish et al., 2010). A second comparable challenge study showed that the percentage of abnormal lung surface ranged from $0.4 \%$ to $39 \%$, with a mean of $14.5 \% \pm 11.6$, relatively similar to the $10 \%$ observed in the current study (Reinhold et al., 2002).

Isolation of $P$. multocida as the principal bacterium recovered from pneumonic lungs at necropsy is considered more indicative of a causal relationship than simple isolation from nasal swabs (Griffin, 2010). In the current study, combined bacteriological findings from DNPS and lung tissue samples support that $P$. multocida was responsible for the development of bacterial bronchopneumonia among study calves. Pasteurella multocida is often isolated from nasal secretions and deep pharyngeal swabs in young calves, and it has been reported that isolation rates in clinically normal cattle 
Table 4. Proportion of calves culture positive on deep nasopharyngeal swab by experimental treatment group: treatment $(\mathrm{TX}) \mathrm{n}=17$, control $(\mathrm{CON}) \mathrm{n}=11$

\begin{tabular}{|c|c|c|c|c|c|c|c|c|}
\hline \multirow{2}{*}{ Bacterial species } & \multicolumn{8}{|c|}{ Deep nasopharyngeal swab number ${ }^{1}$} \\
\hline & \multicolumn{2}{|c|}{1} & \multicolumn{2}{|c|}{2} & \multicolumn{2}{|c|}{3} & \multicolumn{2}{|c|}{4} \\
\hline Pasteurella multocida, no. (\%) & $2(12)$ & $1(9)$ & $2(12)$ & $1(9)$ & $7(41)$ & $4(36)$ & $14(82)$ & $8(73)$ \\
\hline Mannheimia haemolytica, no. (\%) & $1(6)$ & $0(0)$ & $6(35)$ & $5(45)$ & $8(47)$ & $5(45)$ & $5(29)$ & $5(45)$ \\
\hline Trueperella pyogenes, no. (\%) & $2(12)$ & $1(9)$ & $1(6)$ & $1(9)$ & $1(6)$ & $0(0)$ & $1(6)$ & $2(18)$ \\
\hline
\end{tabular}

${ }^{1}$ Swab 1: collected upon arrival to livestock laboratory, $7 \mathrm{~d}$ before challenge. Swab 2: collected $1 \mathrm{~d}$ before challenge (baseline, $\mathrm{d}-1$ ). Swab 3: collected before treatment when at least $1 \mathrm{~cm}^{2}$ of consolidation was observed and $\geq 6 \mathrm{~h}$ had elapsed since challenge. Swab 4: collected following final examination on d 14, before euthanasia.

can be as high as 60\% (Dabo et al., 2007). As shown by our data, clinically normal calves can have subclinical bronchopneumonia; therefore, isolation rates for pathogens in apparently healthy cattle should be interpreted with caution unless lung US is used to confirm that the lungs are ultrasonographically healthy. Additionally, it has been documented that not all clinical cures are due to antibiotic administration (DeDonder and Apley, 2015). A certain level of clinical spontaneous recovery is expected due to fully competent and successful immune response (DeDonder and Apley, 2015). We must keep in mind, however, that clinical spontaneous recovery does not equal bacteriologic cure within the parenchyma of the lung. Our results show disparity in the proportion of calves with clinical respiratory disease at the end of the study compared with those with US consolidation, meaning that calves seemingly free from clinical disease may not actually be recovered.

In a recent study investigating attitude score and respiratory disease, using both lung US and clinical respiratory scoring, calves with clinical BRD were more likely to have depressed attitude than calves with subclinical or no BRD (Cramer, 2018). However, the sensitivity of attitude score alone was poor, at $23 \%$ (Cramer, 2018). Such studies highlight the difficulty of using typical "sickness behavior" of depressed attitude, fever, and increased respiratory rate alone to diagnose bronchopneumonia in preweaned dairy calves. Our study highlights the challenge of identifying the point at which to treat with antibiotics using clinical signs alone. This is emphasized by the fact that respiratory disease developed shortly after infection, as determined by lung consolidation on US, but the onset of clinical signs was variable.

The recurrence of US lung lesions documented in this study might have resulted from a failure to eliminate the initial bacterial lung infection, as indicated by the lack of treatment effects on the final DNPS samples and lung tissue samples. It is plausible that once antibiotic therapy was withdrawn, bacterial replication resumed, ultimately leading to treatment failure. In this case, treatment failure could be defined as the presence of consolidated lung and viable pathogen diagnosed during PME. Our study design eliminated common causes of treatment failures such as chronic cases, inappropriate antibiotic spectrum, antibiotic resistance, and poor compliance. It is more probable that suboptimal antibiotic dose, duration, or frequency of administration contributed to treatment failures in this study.

In general, there is a paucity of data regarding the optimal duration of antibiotic therapy for BRD. Human pneumonia studies evaluating antimicrobial duration

Table 5. Proportion of calves PCR positive on deep nasopharyngeal swab by experimental treatment group: treatment $(\mathrm{TX}) \mathrm{n}=17$, control $(\mathrm{CON}) \mathrm{n}=11$

Deep nasopharyngeal swab number ${ }^{1}$

\begin{tabular}{|c|c|c|c|c|c|c|c|c|}
\hline \multirow{2}{*}{ Item } & & & & & & & & \\
\hline & \multicolumn{2}{|c|}{1} & \multicolumn{2}{|c|}{2} & \multicolumn{2}{|c|}{3} & \multicolumn{2}{|c|}{4} \\
\hline Mannheimia haemolytica, no. (\%) & $3(18)$ & $0(0)$ & $4(24)$ & $4(36)$ & $7(41)$ & $6(55)$ & $7(41)$ & $4(36)$ \\
\hline Mycoplasma bovis, no. (\%) & $0(0)$ & $0(0)$ & $0(0)$ & $1(9)$ & $0(0)$ & $0(0)$ & $0(0)$ & $0(0)$ \\
\hline Coronavirus, no. (\%) & $0(0)$ & $0(0)$ & $0(0)$ & $0(0)$ & $0(0)$ & $1(9)$ & $8(47)$ & $8(73)$ \\
\hline
\end{tabular}

${ }^{1}$ Swab 1: collected upon arrival to livestock laboratory, $7 \mathrm{~d}$ before challenge. Swab 2: collected $1 \mathrm{~d}$ before challenge (baseline, $\mathrm{d}-1$ ). Swab 3: collected before TX when at least $1 \mathrm{~cm}^{2}$ of consolidation was observed and $\geq 6 \mathrm{~h}$ had elapsed since challenge. Swab 4: collected following final examination on d 14, before euthanasia. 
Table 6. Proportion of calves culture positive on lung tissue by experimental treatment group: treatment $(\mathrm{TX}) \mathrm{n}=17$, control $(\mathrm{CON})$ $\mathrm{n}=11$

\begin{tabular}{lccc}
\hline Item & TX & CON & $P$-value \\
\hline Pasteurella multocida, no. (\%) & $13(76)$ & $10(91)$ & 0.62 \\
Mannheimia haemolytica, no. (\%) & $1(6)$ & $0(0)$ & 1.00 \\
Trueperella pyogenes, no. (\%) & $4(24)$ & $2(18)$ & 1.00 \\
Mycoplasma bovis, no. (\%) & $0(0)$ & $0(0)$ & 1.00 \\
\hline
\end{tabular}

have consistently been unable to demonstrate differences between shorter and longer treatment durations (Apley, 2015; Shaddock and Feldman, 2016). In human medicine, the emphasis on duration of therapy is driven by the need to balance therapeutic efficacy with minimizing exposure, in an attempt to curb the development of antibiotic resistance. The label for ampicillin trihydrate indicates that dairy cattle should be treated at a dose of 4.4 to $11 \mathrm{mg} / \mathrm{kg}$ i.m. every $24 \mathrm{~h}$. Treatment should continue 2 to $3 \mathrm{~d}$ after the animal becomes afebrile but no longer than $7 \mathrm{~d}$. In accordance with the human literature and what is practical on farm, we chose to treat calves for a short 3-d duration, with the ability to continue treatment for an additional $2 \mathrm{~d}$ if the calves remained febrile $\left(>39.7^{\circ} \mathrm{C}\right)$ and displayed severe clinical signs of respiratory disease, such as moderate to severe nasal or ocular discharge or spontaneous cough $24 \mathrm{~h}$ past the last treatment. A 24-h post-treatment interval has classically been assumed for antibiotics requiring daily administration for $\mathrm{BRD}$, such as ampicillin trihydrate (Apley, 2015). None of the calves in the TX group were eligible for prolonged therapy based on these guidelines.

For the purposes of this study, a dose of ampicillin at the middle of the label range $(6.6 \mathrm{mg} / \mathrm{kg})$ was used to determine whether judicious antibiotic use would still lead to clinical and US improvement and ultimately bacteriologic cures. For time-dependent antibiotics, such as ampicillin, the most significant factor determining their efficacy is the length of time that serum concentrations exceed the MIC of a given pathogen (Giguére et al., 2013). Increasing the concentration of the drug severalfold above the MIC does not significantly increase the rate of microbial killing; however, more frequent dosing that will ensure adequate coverage throughout a $24-\mathrm{h}$

Table 7. Proportion of calves PCR positive on lung tissue by experimental treatment group: treatment $(\mathrm{TX}) \mathrm{n}=17$, control $(\mathrm{CON})$ $\mathrm{n}=11$

\begin{tabular}{lrcc}
\hline Item & TX & CON & $P$-value \\
\hline Pasteurella multocida, no. (\%) & $14(82)$ & $10(91)$ & 1.00 \\
Mannheimia haemolytica, no. (\%) & $7(41)$ & $1(9)$ & 0.10 \\
Coronavirus, no. (\%) & $7(41)$ & $9(82)$ & 0.05 \\
\hline
\end{tabular}

period may increase the bactericidal activity (Giguére et al., 2013). An early study looking at the bioavailability and pharmacokinetics of ampicillin trihydrate in healthy ruminant calves at a dose of $7.7 \mathrm{mg} / \mathrm{kg}$ i.m. revealed a maximum plasma concentration of $3.7 \mu \mathrm{g} /$ $\mathrm{mL}$ approximately $1 \mathrm{~h}$ after administration, with a halflife of $3.8 \mathrm{~h}$ (Nouws et al., 1982). If we extrapolate this data to our study, where MIC $\leq 0.25 \mu \mathrm{g} / \mathrm{mL}$ for P. multocida, the plasma concentration of ampicillin would have dropped below the MIC in approximately $15 \mathrm{~h}$. The purpose of our study was not to assess the pharmacokinetic properties of ampicillin, but it does emphasize that further research is needed to determine whether increasing the dose or frequency of ampicillin administration could lead to more sustained lung health. Perhaps if we used the highest label dose and based duration of therapy on US resolution of consolidation, as opposed to clinical respiratory signs, we would have had better treatment success.

At the current time, producers are still relying on clinical signs alone for determining the need to treat respiratory disease with antibiotics. Using lung US to make treatment decisions will only be broadly applicable or economically feasible in a field setting with increased involvement from veterinarians trained in lung US. In addition, academic and pharmaceutical-industry research on appropriate dosing regimens for antibiotics used to treat respiratory disease could be greatly enhanced by incorporating lung US into study designs.

A limitation of our study was that we did not evaluate respiratory rate or character as part of our clinical examination. Although significant increases in respiratory rates have been consistently found in previous $P$. multocida challenge studies, results have been mixed regarding how this correlates with pathological and morphological changes in the lung (Reinhold et al., 2002; Dagleish et al., 2010). Respiratory rate analysis would have been more subjective in our population of calves, given that they were group housed and we restrained all calves for respiratory scoring. Handling the calves increased their activity level and may have falsely elevated their respiratory rates. Respiratory rate analysis would have been more beneficial if taken from outside the pen while the calves were resting. Another limitation was that coronavirus was detected in calves on the last day of the study in both DNPS and lung tissue. Respiratory BoCV is associated with mild respiratory disease (coughing, rhinitis) or interstitial pneumonia in 2- to 6-month-old calves (Saif, 2010). Respiratory coinfections and immunosuppression are potential cofactors that can enhance virus shedding (Saif, 2010). It is possible that some of our study calves may have been infected with BoCV and shedding virus below detectable levels when tested before challenge, and the stress 
of the P. multocida pneumonia stimulated increased shedding of viral particles.

An aim of this study was to improve our understanding of bovine lung response to antibiotic therapy after a bacterial respiratory infection, but the calf challenge model limits the generalizability of our results. The pathophysiology of respiratory disease in calves versus adults may vary due to differences in immune responsiveness, pathogen load, and environmental conditions. Although our results provide valuable insight into the effect of antibiotic treatment for BRD in preweaned calves, field trials are warranted to determine the effect of antibiotic treatment in adult dairy cattle with respiratory disease.

\section{CONCLUSIONS}

In this study, respiratory disease was induced with a common respiratory pathogen, P. multocida, and antibiotic treatment was initiated based on the presence of US lung consolidation. Short-term benefits of antibiotic treatment and evidence of relapse were successfully measured using lung US despite inconsistent clinical disease. Our results highlight the disconnect between systemic and pulmonary manifestations of bacterial respiratory disease, and reinforce the need for sensitive diagnostic tools, such as lung US, to accurately classify disease regardless of clinical signs. Opportunity exists to use this technique for early detection of pneumonia, as well as to define recovery from respiratory disease in both the farm and research setting. Future clinical trials should investigate whether adjusting the dose, duration, or frequency of antibiotic administration may lead to more prolonged and sustained lung health and, therefore, more prudent drug use.

\section{ACKNOWLEDGMENTS}

Financial support for this study was provided by Boehringer Ingelheim Vetmedica (Duluth, GA). Special thanks to our dedicated research assistants, the University of Wisconsin-Madison Livestock Laboratory staff, and necropsy technicians at the Wisconsin Veterinary Diagnostic Laboratory (Madison).

\section{REFERENCES}

Apley, M. D. 2015. Treatment of calves with bovine respiratory disease: duration of therapy and posttreatment intervals. Vet. Clin. North Am. Food Anim. Pract. 31:441-453. https://doi.org/10 .1016/j.cvfa.2015.06.001.

Barrett, D. C. 2000. Cost-effective antimicrobial drug selection for the management and control of respiratory disease in European cattle. Vet. Rec. 146:545-550. https://doi.org/10.1136/vr.146.19.545.
Cramer, M. C. 2018. Determining differences in growth, behavior, and serotonin in dairy calves affected by respiratory disease as diagnosed by lung ultrasound and a clinical respiratory score. PhD thesis, Department of Dairy Science, University of Wisconsin, Madison.

Dabo, S. M., J. D. Taylor, and A. W. Confer. 2007. Pasteurella multocida and bovine respiratory disease. Anim. Health Res. Rev. 8:129-150. https://doi.org/10.1017/S1466252307001399.

Dagleish, M. P., J. Finlayson, C. Bayne, S. MacDonald, J. Sales, and J. C. Hodgson. 2010. Characterization and time course of pulmonary lesions in calves after intratracheal infection with Pasteurella multocida A:3. J. Comp. Pathol. 142:157-169. https://doi.org/10 .1016/j.jcpa.2009.10.015.

DeDonder, K. D., and M. D. Apley. 2015. A review of the expected effects of antimicrobials in bovine respiratory disease treatment and control using outcomes from published randomized clinical trials with negative controls. Vet. Clin. North Am. Food Anim. Pract. 31:97-111. https://doi.org/10.1016/j.cvfa.2014.11.003.

Giguére, S., J. F. Prescott, and P. M. Dowling. 2013. The pharmacodynamics of antimicrobial agents. Pages 82-84 in Antimicrobial Therapy in Veterinary Medicine. 5th ed. S. Giguére, J. F. Prescott, and P. M. Dowling, ed. Wiley-Blackwell, Danvers, MA.

Griffin, D. 2010. Bovine pasteurellosis and other bacterial infections of the respiratory tract. Vet. Clin. North Am. Food Anim. Pract. 26:57-71. https://doi.org/10.1016/j.cvfa.2009.10.010.

McGuirk, S. M., and S. F. Peek. 2014. Timely diagnosis of dairy calf respiratory disease using a standardized scoring system. Anim. Health Res. Rev. 15:145-147.

Nouws, J. F. M., C. A. M. van Ginneken, P. Hekman, and G. Ziv. 1982. Comparative plasma ampicillin levels and bioavailability of five parenteral ampicillin formulations in ruminant calves. Vet. Q. 4:62-71. https://doi.org/10.1080/01652176.1982.9693841.

Ollivett, T. L. 2014. Understanding the diagnosis and risk factors for respiratory disease in dairy calves. PhD thesis, Department of Population Medicine, University of Guelph, Ontario, Canada.

Ollivett, T. L., and S. Buczinski. 2016. On-farm use of ultrasonography for bovine respiratory disease. Vet. Clin. North Am. Food Anim. Pract. 32:19-35. https://doi.org/10.1016/j.cvfa.2015.09.001.

Reinhold, P., B. Rabeling, H. Günther, and D. Schimmel. 2002. Comparative evaluation of ultrasonography and lung function testing with the clinical signs and pathology of calves inoculated experimentally with Pasteurella multocida. Vet. Rec. 150:109-114. https: //doi.org/10.1136/vr.150.4.109.

Saif, L. J. 2010. Bovine respiratory coronavirus. Vet. Clin. North Am. Food Anim. Pract. 26:349-364. https://doi.org/10.1016/j.cvfa .2010.04.005.

Scott, P. R. 2013. Clinical presentation, auscultation recordings, ultrasonographic findings and treatment response of 12 adult cattle with chronic suppurative pneumonia: case study. Ir Vet J. 66:5. https://doi.org/10.1186/2046-0481-66-5.

Shaddock, E. J., and C. Feldman. 2016. Shorter antibiotic courses in community-acquired pneumonia-Ready for prime time. J. Thorac. Dis. 8:E1628-E1631. https://doi.org/10.21037/jtd.2016.12.52.

Smith, B. P. 2015. Alterations in body temperature. Pages 33-34 in Large Animal Internal Medicine. 5th ed. B. P. Smith, ed. Elsevier, St. Louis, MO.

Tyler, J. W., D. D. Hancock, S. M. Parish, D. E. Rea, T. E. Besser, S. G. Sanders, and L. K. Wilson. 1996. Evaluation of 3 assays for failure of passive transfer in calves. J. Vet. Intern. Med. 10:304-307. https://doi.org/10.1111/j.1939-1676.1996.tb02067.x.

USDA (United States Department of Agriculture). 2018. Dairy 2014: Health and Management Practices on U.S. Dairy Operations, 2014. USDA-APHIS-VS-CEAH-NAHMS, Fort Collins, CO.

White, T. L., and D. A. Moore. 2009. Reasons for whole carcass condemnations of cattle in the United States and implications for producer education and veterinary intervention. J. Am. Vet. Med. Assoc. 235:937-941. https://doi.org/10.2460/javma.235.8.937. 